\title{
Mobility Tracking for Mobile Ad Hoc Networks
}

\author{
Hui Xu, Min Meng, Jinsung Cho*, Brian J. d'Auriol, and Sungyung Lee \\ Department of Computer Engineering \\ Kyung Hee University, Yongin 449-701, Korea \\ xuhui@oslab.khu.ac.kr
}

\begin{abstract}
In mobile ad hoc networks (MANETs), nodes mobility cause network topologies to change dynamically over time, which complicates important tasks such as broadcasting and routing. Mobility tracking is the task to determine a trajectory of the mobile node in time which can facilitate the forwarding decision in network protocols' design. In this paper, we investigate the regularity of mobility patterns and propose comprehensive mobility predication models, that is, not only piecewise linear but also nonlinear models which are based on nodes' historical location/speed information. As for historical information, we consider not only periodical update but also conditional update networks. Simulation results validate the accuracy of our proposed tracking schemes. We also compare the performance of those schemes and observe their relationship with parameters of update protocols.
\end{abstract}

\section{Introduction}

Mobile ad hoc networks (MANETs) are composed of possibly mobile devices such as sensors, laptops, or PDAs. The absence of a fixed infrastructure in MANETs makes them suitable for applications such as military battlefields, disaster relief and emergency situations. However, the mobility of nodes leads to dynamic network topology changes which complicates important network tasks such as efficient broadcasting and routing.

In most existing localized protocols for MANETs, each node emits "Hello" messages to advertise its presence and update its information. The update protocol can be classified into two kinds: periodical update and conditional update when there is considerable direction change. In periodical update, "Hello" intervals at different nodes can be asynchronous to reduce message collision. Each node extracts neighborhood information from received "Hello" messages to construct its local view for network topology and make decisions based on this view. However, when consider a general case where broadcasting or routing occurs within "Hello" message intervals, while nodes move during this interval, forward decisions will be based on outdated network topology.

Forward decisions based on outdated network topology view may be inaccurate and hence cause delivery failure which can induce poor coverage of broadcast task or route failures. The left part of Fig.1 represents the view of node $i$, and

\footnotetext{
* Corresponding author.
} 


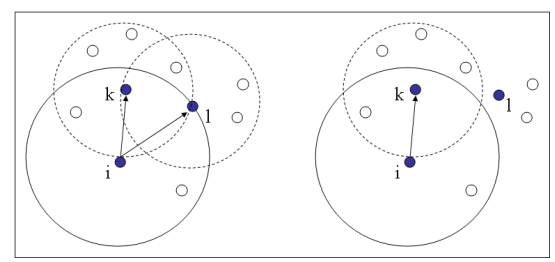

Fig. 1. Impact of outdated topology view on delivery

right part is the real physical topology. Based on an inaccurate local view, node $i$ selects forward nodes $k$ and $l$ and assigns forward nodes' transmission radiuses. However, in real physical topology, node $l$ moves out of the transmission range of $i$ and can't receive the message and forward it. If the dynamics of the network topology could be predicted in advance, appropriate forward decision can be made in order to avoid or reduce delivery failures. In ad hoc networks, the network topology dynamics can be inferred from the mobility of the nodes. Mobility tracking is the task to determine a trajectory of the mobile nodes position in time. Therefore, it could be of significant benefit to the design of network protocols.

We employ here proactive mobility management solutions, mobility prediction, to address the above outdated view problems. We propose both piecewise linear and nonlinear prediction models which make use of node's historical information to predict a trajectory of the mobile nodes position.

The main contributions of this paper are as follows:

- Propose piecewise linear and nonlinear mobility tracking schemes.

- Conduct a simulation study to verify the accuracy of our proposed schemes.

- Compare the performance of these mobility tracking schemes and observe the relationship between the tracking accuracy and update protocols parameters.

The remainder of this paper is organized as follows: Section 2 presents related work and the motivation of our work. In Section 3, we propose the mobility tracking methods based on historical information. Section 4 shows simulation work and results. In Section 5, we conclude this paper.

\section{Related Work}

The node's mobility has a great impact on the performance and capacity of mobile ad hoc networks as discussed in [1].

Much work on mobility management has been done for the design of routing protocols. In the work of Su et al. 2, location information is used to estimate the expiration time of the link between two adjacent hosts which determines the selection of route path. In [3] an overview of existing mobility prediction schemes is given. However, those predictions are also for link availability and path reliability estimation. 
Very little work has been tried to maintain an accurate topology view to assist the route path selection. There are two existing works: one is [4, where a stable zone and a caution zone of each node have been defined based on a node's position, speed, and direction information obtained from GPS. Specifically, a stable zone is the area in which a mobile node can maintain a relatively stable link with its neighbor nodes. A caution zone is the area in which a node can maintain an unstable link with its neighbor nodes. Another is that Wu and Dai (5]) proposed a conservative "two transmission radius" method to compensate the outdated topology local view. However, all of above approaches are passive since they just try to compensate the inaccuracy of the network topology view rather than to predict mobile nodes' positions (mobility tracking) to construct a network topology view in time.

Few mobility tracking for MANETs has been done. One existing work is Zaidi et al. 6. A first-order autoregressive (AR-1) model is used as mobility model. Each node uses an extended Kalman filter to estimate its own mobility state by incorporating network-based signal measurements, such as received signal strength indicators (RSSI) and time-of-arrival (TOA), and the position estimates of the neighbor nodes. The problem of their work is based on special AR-1 model rather than widely used mobility models, which is not suitable to assist the design of network protocols.

\section{Proposed Method}

In this section we first present two update protocols and then propose mobility tracking schemes based on historical information extracted from updates.

\subsection{Update Protocols}

In MANETs the update protocol [7] can be classified into periodical update with fixed time interval and conditional update when there is considerable direction change in a node's motion velocity or direction.

Conditional Update. Suppose that the periodic check for a particular node occurs at time $t_{c}$ with actual location at $\left(x_{c}, y_{c}, z_{c}\right)$. Further suppose that its own most recent update was generated at time $t_{h}$ with location $\left(x_{h}, y_{h}, z_{h}\right)$, speed $v$ and direction $\left(d_{x}, d_{y}, d_{z}\right)$. Then expected location $\left(x_{e}, y_{e}, z_{e}\right)$ at $t_{c}$ can be calculated as

$$
\left\{\begin{array}{l}
x_{e}=x_{h}+\left(t_{c}-t_{h}\right) \cdot v \cdot d_{x} \\
y_{e}=y_{h}+\left(t_{c}-t_{h}\right) \cdot v \cdot d_{y} \\
z_{e}=z_{h}+\left(t_{c}-t_{h}\right) \cdot v \cdot d_{z}
\end{array}\right.
$$

Now the node should check whether the deviated distance $D$ is larger than $\delta$, that is, if $\sqrt{\left(x_{e}-x_{c}\right)^{2}+\left(y_{e}-y_{c}\right)^{2}+\left(z_{e}-z_{c}\right)^{2}}>\delta$ update will be generated. This situation is illustrated in Fig. 2 (a). 


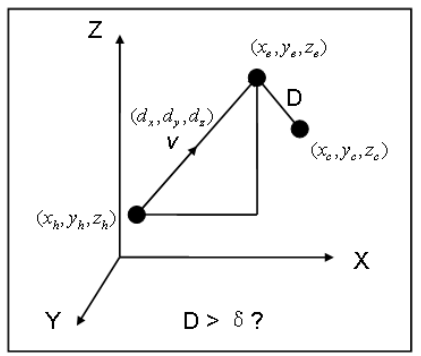

(a) Conditional update

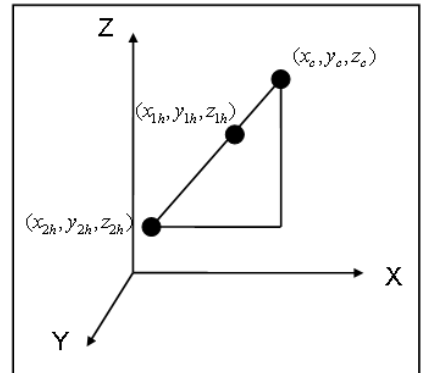

(b) Location-based prediction model

Fig. 2. Update and prediction models sketch

\subsection{Mobility Tracking}

Camp et al. 8 gave a comprehensive survey on mobility models for MANETs, from which we can find that in some models before changing direction nodes move linearly. The other models are not precisely linearly movement, while in a segment view, nodes also move linearly. Therefore we propose two piecewise linear models: location-based and velocity-aided predictions. We also propose one nonlinear model which bases on the principle that in high speed mobility networks we can assume the force acting on the node moving is constant, that is, nodes move with constant acceleration.

Location-based Prediction: Suppose that there are two latest updates records for a particular node respectively at time $t_{2 h}$ and $t_{1 h}\left(t_{2 h}<t_{1 h}\right)$ with location information of $\left(x_{2 h}, y_{2 h}, z_{2 h}\right)$ and $\left(x_{1 h}, y_{1 h}, z_{1 h}\right)$. Assume at least within two successive update periods the node moves in a straight line with fixed speed (depicted in Fig. 2 (b)), we get

$$
\left\{\begin{array}{l}
\frac{x_{2 h}-x_{1 h}}{t_{2 h}-t_{1 h}}=\frac{x_{c}-x_{1 h}}{t_{c}-t_{1 h}} \\
\frac{y_{2 h}-y_{1 h}}{t_{2 h}-t_{1 h}}=\frac{y_{c}-y_{1 h}}{t_{c}-t_{1 h}} \\
\frac{z_{2 h}-z_{1 h}}{t_{2 h}-t_{1 h}}=\frac{z_{c}-z_{1 h}}{t_{c}-t_{1 h}}
\end{array}\right.
$$

then the location $\left(x_{c}, y_{c}, z_{c}\right)$ at a future time $t_{c}$ can be calculated as

$$
\left\{\begin{array}{l}
x_{c}=x_{1 h}+\frac{x_{2 h}-x_{1 h}}{t_{2 h}-t_{1 h}} \times\left(t_{c}-t_{1 h}\right) \\
y_{c}=y_{1 h}+\frac{y_{2 h}-y_{1 h}}{t_{2 h}-t_{1 h}} \times\left(t_{c}-t_{1 h}\right) \\
z_{c}=z_{1 h}+\frac{z_{2 h}-z_{1 h}}{t_{2 h}-t_{1 h}} \times\left(t_{c}-t_{1 h}\right) .
\end{array}\right.
$$

However in conditional update networks since the latest update represents considerable changes compared to previous update, this model cannot be used.

Velocity-aided Prediction: Let $\left(x_{1 h}, y_{1 h}, z_{1 h}\right)$ and $\left(v_{x}^{\prime}, v_{y}^{\prime}, v_{z}^{\prime}\right)$ respectively be the location and velocity of its latest update for a particular node. Assume the 


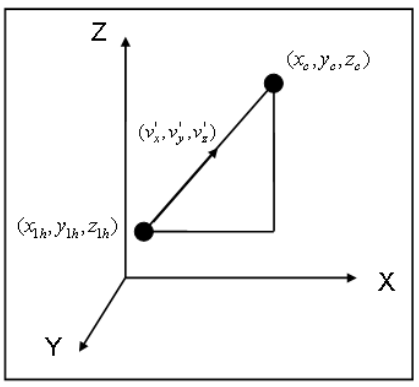

(a) Velocity-aided prediction model

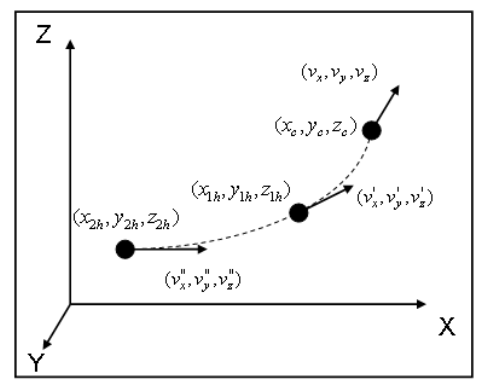

(b) Constant acceleration prediction model

Fig. 3. Prediction models sketch

node moves with that speed within one update period (depicted in Fig. $3(\mathrm{a})$ ), the location $\left(x_{c}, y_{c}, z_{c}\right)$ at a future time $t_{c}$ can be calculated as

$$
\left\{\begin{array}{l}
x_{c}=x_{1 h}+v_{x}^{\prime} \cdot\left(t_{c}-t_{1 h}\right) \\
y_{c}=y_{1 h}+v_{y}^{\prime} \cdot\left(t_{c}-t_{1 h}\right) \\
z_{c}=z_{1 h}+v_{z}^{\prime} \cdot\left(t_{c}-t_{1 h}\right) .
\end{array}\right.
$$

Constant Acceleration Prediction: Let $\left(x_{1 h}, y_{1 h}, z_{1 h}\right)$ and $\left(x_{2 h}, y_{2 h}, z_{2 h}\right)$ be the latest two updates for a particular node at $t_{1 h}$ and $t_{2 h}\left(t_{2 h}<t_{1 h}\right)$. Let $\left(v_{x}^{\prime}, v_{y}^{\prime}, v_{z}^{\prime}\right)$ and $\left(v_{x}^{\prime \prime}, v_{y}^{\prime \prime}, v_{z}^{\prime \prime}\right)$ respectively be the velocity of those two update for a particular node. Now assume to predict the location $\left(x_{c}, y_{c}, z_{c}\right)$ of the node at a future time $t_{c}$ before new update time comes(depicted in Fig. 3 (b)). As all know the principle motion law

$$
V=v+a t
$$

and

$$
S=v t+\frac{1}{2} a t^{2}=\bar{v} t=\frac{v+V}{2} t,
$$

here $S$ is the displacement, $v$ is the initial velocity and $a$ is acceleration during period $t$. We employ $V$ denoting the final velocity after period $t$.

Assume the fixed acceleration is $\left(a_{x}, a_{y}, a_{z}\right)$ and apply above principle to $\mathrm{X}$ dimension, we can get

$$
\left\{\begin{array}{l}
v_{x}^{\prime}=v_{x}^{\prime \prime}+a_{x}\left(t_{2 h}-t_{1 h}\right) \\
v_{x}=v_{x}^{\prime}+a_{x}\left(t_{c}-t_{1 h}\right) \\
x_{c}-x_{1 h}=\frac{\left(v_{x}^{\prime}+v_{x}\right)}{2}\left(t_{c}-t_{1 h}\right)
\end{array}\right.
$$

Then we can get expected location $x_{c}$ as

$$
x_{c}=x_{1 h}+\frac{2 v_{x}^{\prime}+\left(v_{x}^{\prime}-v_{x}^{\prime \prime}\right) \frac{t_{c}-t_{1 h}}{t_{2 h}-t_{1 h}}}{2}\left(t_{c}-t_{1 h}\right) .
$$




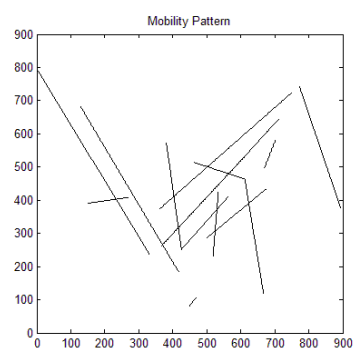

(a)under Random Waypoint model

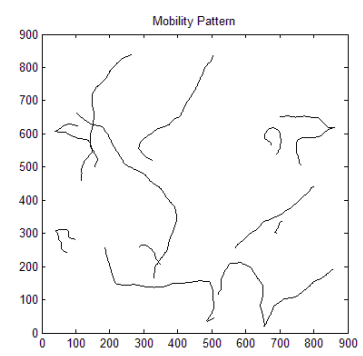

(b) under Gauss-Markov model

Fig. 4. Traveling pattern of MNs

Since $\mathrm{Y}$ and $\mathrm{Z}$ dimensions are the same with $\mathrm{X}$-dimension, finally we have

$$
\left\{\begin{array}{l}
x_{c}=x_{1 h}+\frac{2 v_{x}^{\prime}+\left(v_{x}^{\prime}-v_{x}^{\prime \prime}\right) \frac{t_{c}-t_{1 h}}{t_{2 h}-t_{1 h}}}{2}\left(t_{c}-t_{1 h}\right) \\
y_{c}=y_{1 h}+\frac{2 v_{y}^{\prime}+\left(v_{y}^{\prime}-v_{y}^{\prime \prime}\right) \frac{t_{c}-t_{1 h}}{t_{2 h}-t_{1 h}}}{2}\left(t_{c}-t_{1 h}\right) \\
z_{c}=z_{1 h}+\frac{2 v_{z}^{\prime}+\left(v_{z}^{\prime}-v_{z}^{\prime \prime}\right) \frac{t_{c}-t_{1 h}}{t_{2 h}-t_{1 h}}}{2}\left(t_{c}-t_{1 h}\right) .
\end{array}\right.
$$

Above we proposed mobility tracking schemes to predict nodes' trajectories. However, there exist several possible imprecision factors: GPS reading obtained may not always be accurate due to various reasons (e.g., multi-path fading, indoor conditions, etc.); node suddenly changes its direction before future prediction time; the movement speed of node can accelerate or decelerate; node moves nonlinearly (only for piecewise linear models). In the real world, these factors cause predicted trajectories inaccurate.

\section{Performance Evaluation}

In this section, we evaluate the performance of our mobility tracking schemes and the effect of update protocols on them.

\subsection{Simulation Environment}

We use ns-2.28 [9] and its CMU wireless extension as the simulation tool. To demonstrate the comprehensive effectiveness of our prediction proposals, we do experiments in both linear (Random Waypoint) and nonlinear (Gauss-Markov) mobility models [8] which are widely used in simulating protocols designed for MANETs. Fig. 4 shows the traveling pattern of MNs using those two mobility models. Table 1 displays our mobility tracking simulation parameters. 
Table 1. Simulation parameters for mobility tracking

\begin{tabular}{|c|c|}
\hline Parameters & Value \\
\hline Simulation network size and nodes number & $900 \times 900 \mathrm{~m}^{2}$ and 12 \\
\hline Mobile nodes speed range & {$[0,15] \mathrm{m} / \mathrm{s}$} \\
\hline Simulation time & $150 \mathrm{~s}$ \\
\hline Location record/check interval & $2 \mathrm{~s}$ \\
\hline Prediction interval & $20 \mathrm{~ms}$ \\
\hline Reference distance for conditional update & $5 \mathrm{~m}$ \\
\hline
\end{tabular}

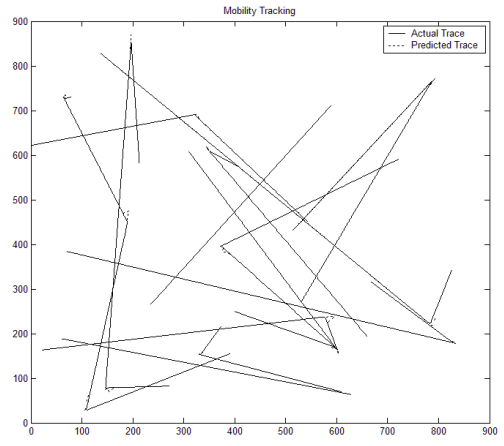

(a)under Random Waypoint model

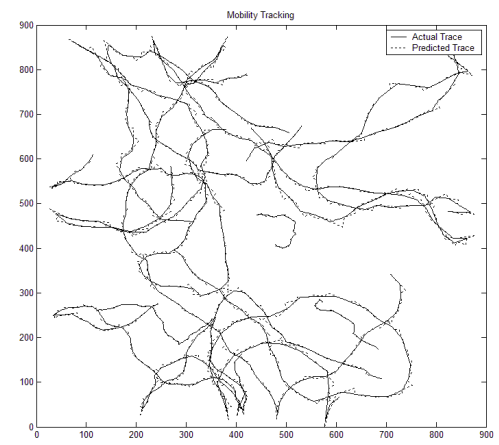

(b) under Gauss-Markov model

Fig. 5. Example of mobility tracking in periodical update case

\subsection{Evaluation of Mobility Tracking}

A sample simulation result with location-based prediction under periodical update is illustrated in Fig. 5. We can see that our mobility tracking scheme performs well, especially under Random Waypoint Mobility Model.

We use root mean square prediction error ( $R M S E$ ) as a metric to evaluate the accuracy of mobility prediction models. Suppose there are $N$ nodes in simulation networks and for any node $j$, assume there are $K$ prediction points for it and let $x_{i}, y_{i}, z_{i}$ represent the actual location for the $i t h$ point and $x_{i}^{\prime}, y_{i}^{\prime}, z_{i}^{\prime}$ be the predicted location with a certain prediction model, then the RMSE for the node $\left(E_{j}\right)$ can be calculated as

$$
\sqrt{\frac{1}{K} \sum_{i=1}^{K}\left[\left(x_{i}^{\prime}-x_{i}\right)_{2}+\left(y_{i}^{\prime}-y_{i}\right)^{2}+\left(z_{i}^{\prime}-z_{i}\right)^{2}\right]}
$$




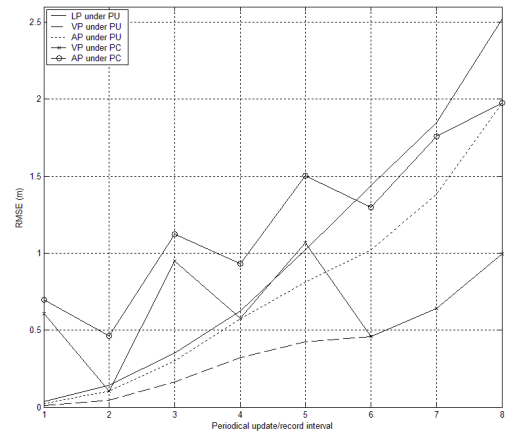

(a)under Random Waypoint model

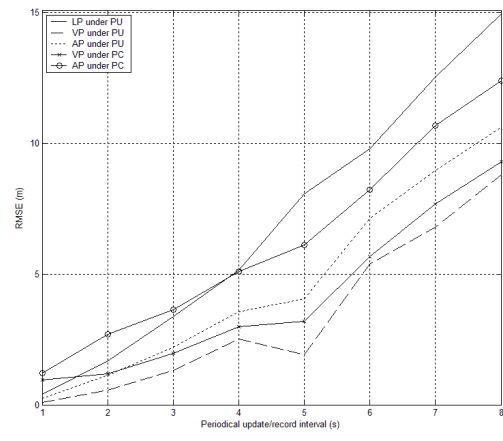

(b) under Gauss-Markov model

Fig. 6. The accuracy of prediction models with various record/check intervals

Table 2. RMSE of mobility prediction schemes under Random Waypoint Model

\begin{tabular}{c|c|c}
\hline \hline Records Type & Prediction Scheme & RMSE Value \\
\hline Periodical & Location-based & 0.144803 \\
Record & Velocity-aided & 0.0448978 \\
& Constant Acceleration & 0.103663 \\
\hline Periodical & Velocity-aided & 0.104336 \\
Check & Constant Acceleration & 0.460686 \\
\hline
\end{tabular}

And the RMSE for a prediction model $\left(E_{p}\right)$ will be the mean value

$$
E_{p}=\frac{1}{N} \sum_{j=1}^{N} E_{j} .
$$

The smaller the RMSE, the more accurate the prediction model is.

Table 2 shows the RMSE results of our proposed schemes under the Random Way Point Model. Table 3 shows the RMSE results of our proposed schemes under the Gauss-Markov Model. These tables indicate again that our mobility tracking scheme performs better in Linear Mobility Models than in Nonlinear Mobility Model. Under periodical update protocols, the RMSE of our velocity-aided mobility tracking is smallest and that of location-based tracking is largest, that is, velocity-aided tracking is most accurate and nonlinear tracking is more accurate than location-based scheme. Under conditional update protocols, velocity-aided tracking is yet more accurate than constant acceleration tracking scheme.

To observe the effect of periodical record interval on the tracking performance, Fig. 6 shows the accuracy (RMSE) of prediction models obtained under various periodical record/check intervals. In Fig. 6 LP represents location-based 
Table 3. RMSE of mobility prediction schemes under Gauss-Markov model

\begin{tabular}{c|c|c}
\hline \hline Records Type & Prediction Scheme & RMSE Value \\
\hline Periodical & Location-based & 1.67795 \\
Record & Velocity-aided & 0.564541 \\
& Constant Acceleration & 1.14196 \\
\hline Periodical & Velocity-aided & 1.19953 \\
Check & Constant Acceleration & 2.68946 \\
\hline
\end{tabular}

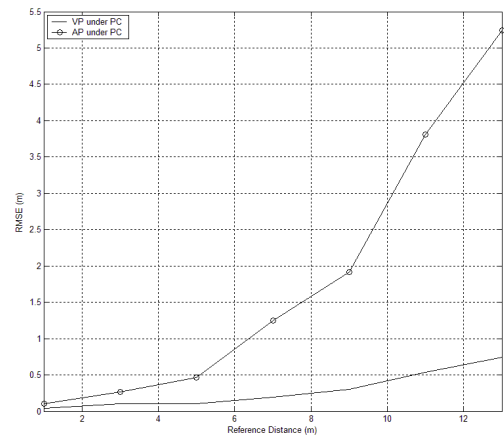

(a)under Random Waypoint model

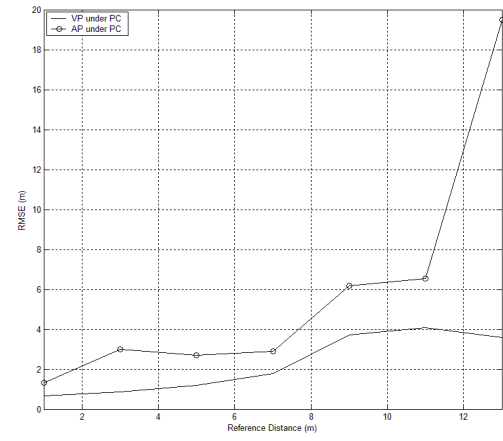

(b) under Gauss-Markov model

Fig. 7. The accuracy of prediction models with various reference distances

prediction; VP represents velocity-aided prediction; AP represents constant acceleration prediction; PU represents periodical update; PC represents periodical check (conditional update). As expected, when the update frequency decreases, the accuracy of the mobility tracking also decreases. Therefore we should choose small enough update interval to guarantee the tracking accuracy.

To observe the effect of reference distance for periodical check on the prediction accuracy, Fig. 7 shows the accuracy (RMSE) of prediction models obtained with various reference distances. Increasing the update reference distance tends to greatly degrade the tracking performance of velocity-based scheme, and under the nonlinear mobility model, the effect is more obvious. But the tracking performance of constant acceleration scheme remains fairly stable.

\section{Conclusions}

In this paper, we proposed both piece-wise linear and constant acceleration mobility tracking schemes to predict nodes' trajectory. Our prediction schemes are based on historical information achieved through periodic or conditional updates. In simulation work, we focus on evaluating the accuracy (RMSE) of our schemes and observe the trend of RMSE under varied update/check interval and 
varied reference distance in conditional update protocols. From the comparison of those schemes, we can see that the velocity-based scheme is best.

\section{Acknowledgment}

This work was supported by grant No. R01-2005-000-10267-0 from Korea Science and Engineering Foundation in Ministry of Science and Technology.

\section{References}

1. Grossglauser, M., Tse, D.: Mobility Increases the Capacity of Ad-Hoc Wireless Networks. IEEE Infocom, Alaska, USA (2001)

2. Su, W., Lee, S.-J., Gerla, M.: Mobility Prediction and Routing in Ad Hoc Wireless Networks. International J. Network Management 11(1), 3-30 (2001)

3. Chellappa Doss, R., Jennings, A., Shenoy, N.: A Review of Current Mobility Prediction Techniques for Ad Hoc Networks. In: The 4th IASTED International MultiConference Wireless And Optical Communications, Banff, Canada, pp. 536-542 (2004)

4. Kim, W.-I., Kwon, D.H., Suh, Y.-J.: A reliable route selection algorithm using local positioning systems in mobile ad hoc networks. In: The IEEE International Conference on Communications, Amsterdam, USA (2001)

5. Wu, J., Dai, F.: Mobility Control and Its Applications in Mobile Ad Hoc Networks. In: Handbook of Algorithms for Wireless Networking and Mobile Computing, 22501-22-518 (2006)

6. Zaidi, Z.R., Mark, B.L.: A Distributed Mobility Tracking Scheme for Ad Hoc Networks Based on an Autoregressive Model. In: The 6th International Workshop of Distributed Computing, Kolkata, India, pp. 447-458 (2004)

7. Shah, S.H., Nahrstedt, K.: Predictive location-based QoS routing in mobile ad hoc networks. In: IEEE International Conference on Communications, New York, USA (2002)

8. Camp, T. (ed.): A Survey of Mobility Models for Ad Hoc Network Research. Wireless Comm. and Mobile Computing, special issue on mobile ad hoc networking: research, trends and applications 2(5), 483-502 (2002)

9. Fall, K., Varadhan, K.: The ns Manual. The VINT Project, UCB, LBL, USC/ISI and Xerox PARC (2002) 